\title{
Immunophenotypic analysis of cerebrospinal fluid reveals concurrent development of ATL in the CNS of a HAM/TSP patient
}

\author{
Reina Takeda ${ }^{1} \cdot$ Tomohiro Ishigaki $^{1,2} \cdot$ Nobuhiro Ohno $^{1,3} \cdot$ Kazuaki Yokoyama $^{1} \cdot$ Toyotaka Kawamata $^{1,3}$. \\ Tomofusa Fukuyama $^{1,4} \cdot$ Natsumi Araya $^{5} \cdot$ Yoshihisa Yamano $^{5} \cdot$ Kaoru Uchimaru ${ }^{1,6} \cdot$ Arinobu Tojo $^{1,2,3}$
}

Received: 9 September 2019 / Revised: 26 December 2019 / Accepted: 26 December 2019 / Published online: 13 January 2020

(c) Japanese Society of Hematology 2020

\begin{abstract}
Both adult T-cell leukemia/lymphoma (ATL) and human T-cell leukemia virus type 1 (HTLV-1)-associated myelopathy/ tropical spastic paraparesis (HAM/TSP) can be induced by HTLV-1, but concurrent development has been rarely reported. We present the case of a 55-year-old female who developed cranial nerve symptoms after a 20-year history of HAM/TSP. Although multiple white matter lesions were observed on brain magnetic resonance imaging, no abnormalities were seen on a systemic computed tomography scan. Quantitative flow-cytometric analysis of cell populations in the cerebrospinal fluid (CSF) revealed that most of the infiltrating cells were not inflammatory cells, but HTLV-1-infected CD4 ${ }^{+}$CADM$1^{+} \mathrm{T}$-cells completely lacking CD7 expression. As stepwise downregulation of CD7 is correlated with disease progression from HTLV-1 carrier to aggressive ATL, the CSF cells were classified as aggressive ATL; these cells exhibited a more progressed phenotype than those in peripheral blood (PB). HAM/TSP disease activity was estimated to be low. From these and other examinations, we made a diagnosis of acute-type ATL, which unusually developed in the central nervous system at initial onset prior to systemic progression. In ATL cases with a challenging diagnosis, immunophenotypic characterization of CSF and PB is valuable for differential diagnosis and understanding disease status.
\end{abstract}

Keywords Adult T-cell leukemia/lymphoma (ATL) · HTLV-1 associated myelopathy/tropical spastic paraparesis (HAM/ TSP) $\cdot$ Multi-color flow cytometry $\cdot$ Central nervous system involvement

Tomohiro Ishigaki

ishigaki@ims.u-tokyo.ac.jp

1 Department of Hematology/Oncology, Research Hospital, The Institute of Medical Science, The University of Tokyo, 4-6-1 Shirokanedai, Minato-ku, Tokyo 108-8639, Japan

2 Department of Laboratory Medicine, Research Hospital, Institute of Medical Science, The University of Tokyo, Tokyo, Japan

3 Division of Molecular Therapy, The Institute of Medical Science, The University of Tokyo, Tokyo, Japan

4 Division of Cellular Therapy, The Institute of Medical Science, The University of Tokyo, Tokyo, Japan

5 Department of Rare Diseases Research, Institute of Medical Science, St. Marianna University School of Medicine, Kawasaki, Kanagawa, Japan

6 Laboratory of Tumor Cell Biology, Department of Computational Biology and Medical Science, Graduate School of Frontier Sciences, The University of Tokyo, Tokyo, Japan

\section{Introduction}

The prevalence of human T-cell leukemia virus type 1 (HTLV-1) infection is estimated to be at least 5-10 million people worldwide [1]. HTLV-1 is transmitted vertically from mother to child via breastfeeding or horizontally by sexual intercourse and contaminated blood products [1]. Although the majority of HTLV-1-infected people remain asymptomatic for life, approximately 0.25 to $3 \%$ of HTLV-1-infected individuals develop HTLV-1-associated myelopathy/tropical spastic paraparesis (HAM/TSP), and another 5\% develop adult T-cell leukemia/lymphoma (ATL) [2]. ATL occurs preferentially in older people with a median age of diagnosis of 68 years and slightly more in men [3]. ATL usually develops after decades of a non-symptomatic incubation period since the first infection, mostly via breastfeeding from an HTLV-1-carrier mother [2, 3]. By contrast, HAM/ TSP occurs three times more often in women as in men and preferentially at around 40 years of age [2]. HAM/TSP can develop with various incubation period lengths from several 
months to 30 years after vertical or horizontal transmission [2]. The CD4 + CD25 + CCR4 + T-cell population, which is generally recognized as a subset that includes regulatory $\mathrm{T}$ (Treg) cells and Th 2 cells, is the predominant viral reservoir of HTLV-1 in both ATL and HAM/TSP [4]. During ATL, the FOXP3 + population, which has Treg-like characteristics immunophenotypically and functionally, is maintained, leading to suppression of normal immunity in favor of tumor proliferation [4]. By contrast, the primary HTLV-1-infected T-cells in patients with HAM/TSP become Th1-like cells with reduced FOXP3 expression that overproduce interferon-gamma, resulting in over-reactive inflammation in the spinal cord $[2,4,5]$. Hence, the host immune response appears different between ATL and HAM/TSP $[4,5]$.

Concurrent development of HAM/TSP and ATL is uncommon, and a few cases of patients with HAM/TSP who developed ATL and vice versa have been reported [6]. Here, we describe an unusual case of aggressive ATL developing in the central nervous system (CNS) of a patient with HAM/ TSP. As the patient lacked typical symptoms and signs associated with ATL, the diagnosis was challenging.

\section{Case presentation}

A 55-year-old female patient with HAM/TSP was referred to our hospital due to somnolence. Bilateral spastic gait disorder progressed gradually during her 20-year history with HAM/TSP. She became wheelchair-dependent at the age of 52. HAM/TSP had been observed and treated with physical therapy for the last several years. Three months before admission, she presented with postural instability, followed by cranial nerve symptoms, such as visual disturbance, hearing loss, and a facial palsy around the mouth. Altered mental status and cognitive impairment also developed 1 month before admission.

On admission, she was drowsy and bedridden with a Glasgow Coma Scale score of 10. A neurological examination revealed spastic tetraplegia, a bilaterally positive Babinski's reflex, and an exaggerated deep tendon reflex in the upper extremities. Loss of the pupillary reflex and horizontal nystagmus were found bilaterally. No neck stiffness or any other meningeal irritation was observed. As these cranial nerve symptoms are not generally observed in HAM/TSP, the concurrence of another intracranial disease was suspected. Except for neurological findings, no abnormal signs including skin lesions were observed on a physical examination. The peripheral blood (PB) examination revealed a white blood cell (WBC) count of $6.7 \times 10^{9} / \mathrm{L}$, a hemoglobin level of $11.3 \mathrm{~g} / \mathrm{dL}$, and a platelet count of $29.9 \times 10^{9} / \mathrm{L}$. While morphologically normal lymphocytes accounted for $20 \%$ of WBCs, atypical lymphocytes were observed in $3.0 \%$ (Fig. 1). C-reactive protein content had

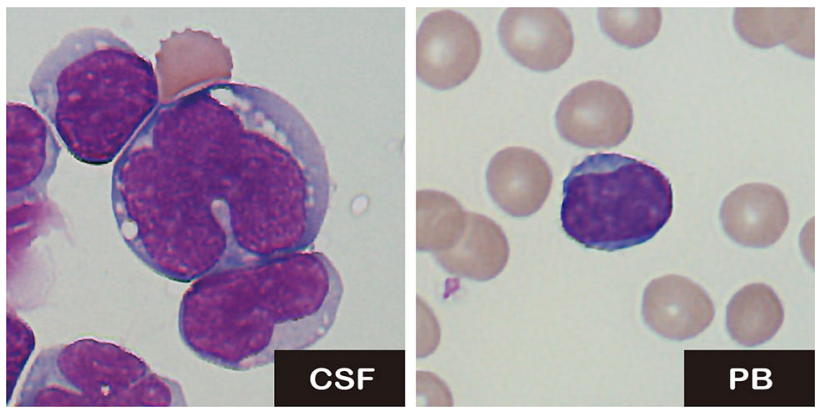

Fig. 1 Morphological characteristics of the lymphocytes in cerebrospinal fluid (CSF) and peripheral blood (PB). Representative images of infiltrating lymphocytes in the CSF (left) and PB (right) are shown (May-Giemsa stain; original magnification, $1000 \times$ ). Most infiltrating cells in the CSF were large abnormal lymphocytes with a vacuolized cytoplasm and a highly cleaved nucleus. By contrast, lymphocytes in the $\mathrm{PB}$ remained slightly atypical and small to medium in size

increased slightly to $0.41 \mathrm{mg} / \mathrm{dL}$, and albumin content had decreased to $2.8 \mathrm{mg} / \mathrm{dL}$. Lactic dehydrogenase (LDH), blood urea nitrogen, and corrected calcium levels remained within the normal limits. The level of soluble interleukin 2 receptor (sIL-2R) was elevated at $1210 \mathrm{U} / \mathrm{mL}$. The serum HTLV-1 antibody test was positive. Lymphadenopathy, hepatosplenomegaly, or any other abnormalities was not detected using systemic computed tomography (CT). Brain magnetic resonance imaging (MRI) revealed that there were multiple lesions in the diffuse area of the bilateral frontal cerebral white matter, the corpus callosum, and the pons. These intracranial lesions were hypo-/isointense in T1-weighted images and hyperintense in T2-weighted images, but they were not enhanced by gadolinium-diethylenetriamine pentaacetate. A cerebrospinal fluid (CSF) test revealed the cell count of $46.2 / \mu \mathrm{L}$ with large abnormal lymphocytes (Fig. 1), protein content of $37.0 \mathrm{mg} / \mathrm{dl}$, glucose content of $9 \mathrm{mg} / \mathrm{dl}$, and LDH content of $66 \mathrm{IU} / \mathrm{L}$. The CSF tested positive for the HTLV-1 antibody. Notably, the level of sIL-2R in the CSF increased remarkably to $7000 \mathrm{U} / \mathrm{mL}$, which was approximately six times as high as the PB level (Table 1). The proviral load of the CSF was also much higher than that of the $\mathrm{PB}$. Polymerase chain reaction (PCR) analysis using the CSF sample revealed that the sample was negative for adenovirus, cytomegalovirus, Epstein-Barr virus, herpes simplex virus, human herpesvirus, varicella zoster virus, Aspergillus, Candida albicans/glabrata, and tuberculosis.

Next, multi-color flow-cytometric analysis of the cell populations in the CSF was performed with absolute enumeration (Fig. 2a-g). There were few neutrophils, monocytes, B-cells, NK-cells, and CD8-positive T-cells in the $\mathrm{CSF}$, which ruled out rapid deterioration resulting from HAM/TSP and infectious intracranial diseases, such as a cerebral abscess, encephalitis, or meningitis. Most of the infiltrating cells were phenotypically homogeneous and 
Table 1 Characteristics of the cerebrospinal fluid (CSF) and peripheral blood (PB) at the time of diagnosis

\begin{tabular}{lll}
\hline Cerebrospinal fluid (CSF) & Peripheral blood (PB) \\
\hline $\begin{array}{l}\text { Large-sized abnormal lymphocytes with } \\
\text { basophilic plasma and strongly cleaved } \\
\text { nuclei }\end{array}$ & Cell appearance & $\begin{array}{c}\text { Small to medium-sized atypical lymphocytes } \\
\text { with basophilic plasma and less cleaved } \\
\text { nuclei }\end{array}$ \\
100.0 & Frequency of atypical and abnormal lympho- & 3.0 \\
66 & cytes $(\% /$ nucleated cells $)$ & 209 \\
37 & LDH (IU/L) & 5.9 \\
9 & Protein $(\mathrm{g} / \mathrm{dL})$ & 77 \\
7000 & Glucose $(\mathrm{mg} / \mathrm{dL})$ & 1210 \\
+ & sIL-2R $(\mathrm{U} / \mathrm{mL})$ & + \\
93.92 & HTLV-1 antibody & 16.48 \\
35 & HTLV-1 proviral load (copy number/100 cells) & Not studied \\
$10,336.9$ & Neopterin $(\mathrm{pmol} / \mathrm{mL})$ & Not studied \\
\hline
\end{tabular}

LDH, Lactic dehydrogenase; sIL-2R, soluble interleukin 2 receptor; HTLV-1, human T-cell leukemia virus type 1

positive for CD3, CD4, CADM-1, and CCR4 (not shown), but negative for CD7. By contrast, a small fraction of the CD4-positive cells in the PB consisted of three different cell populations as seen in a CD7 versus CADM-1 plot (Fig. 2h). As reported previously, CD4 + T-cells that strongly express CADM-1 are HTLV-1-infected cells, and stepwise downregulation of CD7 in HTLV-1-infected cells is associated with disease progression from HTLV-1-carrier status to aggressive ATL [7-10]. The difference in the expression pattern of CD7 and CADM-1 in CD4-positive cells implied a more advanced disease status in the CSF (Fig. 2g, h), and the pattern in the CSF was very similar to that observed in aggressive ATL. Moreover, these infiltrating cells in the CSF were later cytologically categorized as class V. Taken together, the patient was finally diagnosed with acute-type ATL with CNS involvement.

Considering the ATL activity, mainly in the CNS, and safety concerns about giving systemic chemotherapy to the cognitively impaired patient, we decided to precede intrathecal therapy before systemic chemotherapy. The treatment started immediately with repetitive triple intrathecal therapy (methotrexate, cytarabine, and prednisolone). Nevertheless, before administration of systemic chemotherapy, the patient died of central neurogenic respiratory failure on the sixth day of hospitalization. An autopsy was not permitted.

\section{Discussion}

We present a case of aggressive ATL that developed in the CNS without systemic progression. This case was classified as acute-type ATL according to Shimoyama's criteria, and the diagnosis was difficult because the patient did not exhibit typical ATL features that met the criteria, such as $>5 \%$ abnormal lymphocytes, swollen lymph nodes, and skin lesions [11]. CNS invasion is observed in 10-25\% of patients with ATL, and it almost always occurs after rapid systemic progression of ATL [12, 13]. Hence, a case like ours, where CNS involvement was the initial ATL disease presentation and systemic features were lacking, is very rare [13].

Treatment for this patient was decided considering her altered mental status, cognitive impairment, and progressive decrease in consciousness. We assessed systemic chemotherapy was inapplicable at first, and urgent therapeutic intervention for CNS lesions was necessary. Moreover, as no other active ATL lesions were detected, the ATL activity was considered to be mainly in the CNS. Therefore, we started with intrathecal therapy before systemic chemotherapy.

Our patient already had another HTLV-1-induced disease, HAM/TSP, before she developed ATL. Concurrent development of HAM/TSP and ATL is uncommon, and few cases have been reported of patients with HAM/TSP who develop ATL and vice versa [6]. A high proviral load of HTLV-1 is a significant risk for both the progression of HAM and development of ATL [2]. Chronic meningomyelitis with axonal degeneration resulting from HAM/TSP leads to motor and sensory disturbances, but intracranial symptoms rarely occur because HAM/TSP particularly affects the middle to lower thoracic cord [2]. Therefore, the series of cranial nerve symptoms observed in our patient, including visual disturbance, deafness, facial palsy, cognitive impairment, and consciousness disorder were considered to be caused by another disease, which was finally concluded to be CNS involvement of ATL. The survival period from the onset of ATL to death was only 3 months, indicating that ATL had progressed rapidly in the CNS. As the survival period of acute-type ATL is generally short from a few weeks to months without chemotherapy [14], early detection and intervention are important for ATL. When HAM patients 


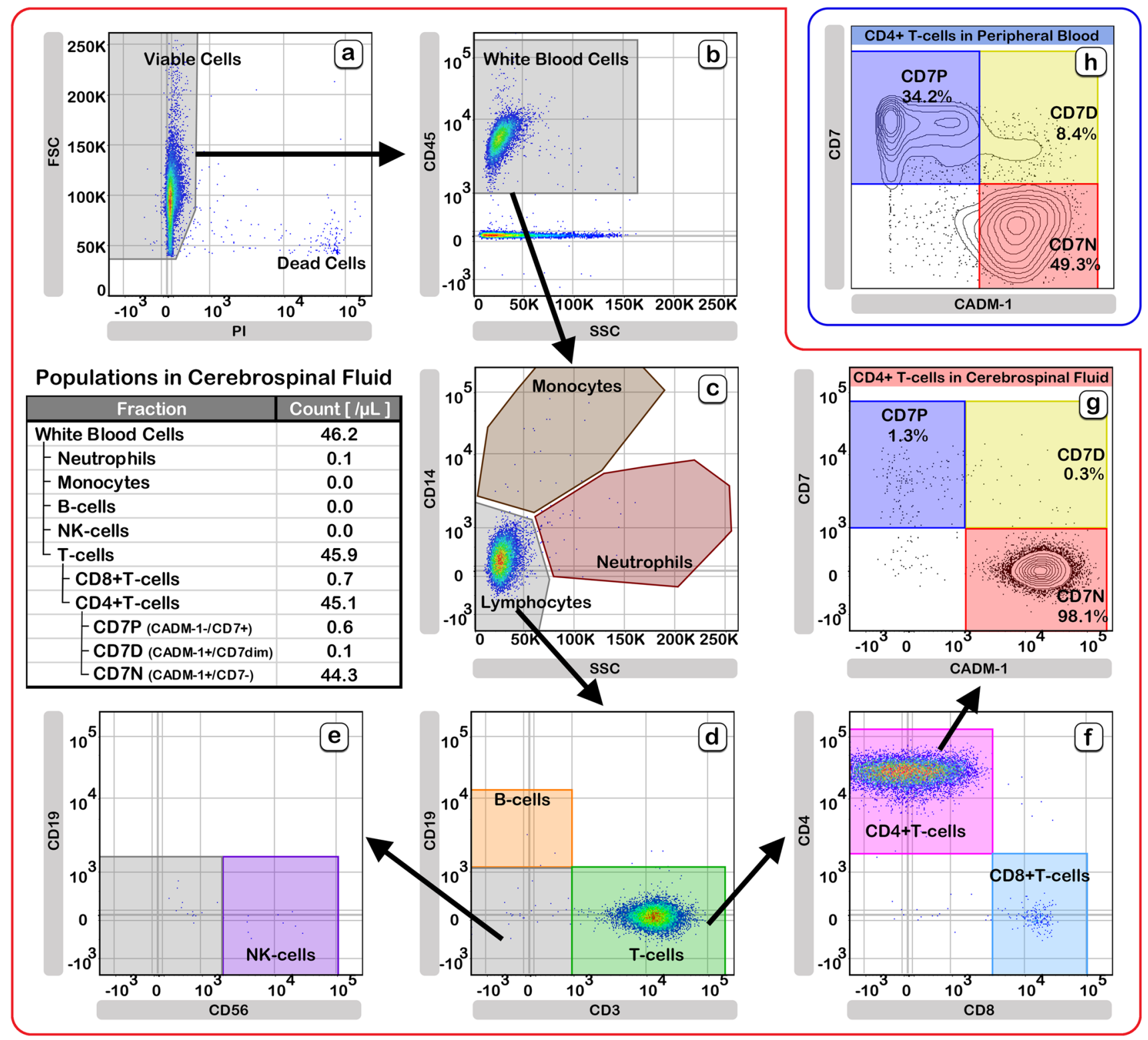

Fig. 2 Quantitative flow-cytometric analysis of cell populations in the cerebrospinal fluid (CSF) and peripheral blood (PB). Cell populations in the CSF were analyzed and quantified using multi-color flow cytometry. Viable cells, white blood cells, neutrophils, monocytes, B-cells, T-cells, NK-cells, CD8-positive T-cells, and CD4-positive T-cells were gated sequentially $(\mathbf{a}-\mathbf{f})$. Then, the expression patterns of CD7 and CADM-1 in CD4-positive T-cells were plotted (g), and the expression profile was compared with CD4-positive T-cells

present with unusual intracranial symptoms, multimodal assessments should be performed for differential diagnosis and evaluation of the disease status.

Several CSF biomarkers have been studied for evaluating disease activity of HAM/TSP. The levels of neopterin and CXCL10 in the CSF are closely correlated with HAM/TSP disease activity $[15,16]$. A previous study demonstrated that the median level of neopterin in the CSF of a deteriorating in the PB (h). Most of the cells in the CSF were positive for CD4 and CADM-1 but negative for CD7. These CD4-positive T-cells in the CSF exhibited an apparently advanced ATL profile compared with those in the $\mathrm{PB}$. Only very small populations of $\mathrm{CD} 4+\mathrm{CD} 7 \mathrm{P}$ cells and CD8 + T-cells, which are the counterparts of uninfected CD4+T-cells and cytotoxic T-lymphocytes respectively, were observed, and no significant infiltration of other cells, such as neutrophils, monocytes/macrophages, B-cells, and NK-cells, was detected

HAM/TSP patient is $20-30 \mathrm{pmol} / \mathrm{mL}$, whereas most stable patients with HAM/TSP exhibited values of $<10 \mathrm{pmol} /$ $\mathrm{mL}$ [15]. CSF CXCL10 is also reported to distinguish between rapid and slow progressors with an optimal cutoff of $4400 \mathrm{pg} / \mathrm{ml}$ [16]. Although these markers are very useful to evaluate HAM/TSP disease activity, discriminating other diseases is difficult. Indeed, our patient presented with higher levels of neopterin and CXCL10 in the CSF, 
although HAM/TSP disease activity seemed to be low. As CSF neopterin is reported to be a potential diagnostic biomarker of primary CNS lymphoma with an optimal cut-off value of $10 \mathrm{pmol} / \mathrm{mL}$ [17], discriminating ATL in the CNS from deterioration resulting from HAM is difficult. Another marker suited for this purpose is CSF sIL-2R. In our case, there was an increased level of sIL-2R in the patient's CSF. According to previous studies, the majority of ATL patients with CNS involvement have elevated levels of sIL-2R in the CSF [18], whereas levels of sIL-2R in the CSF of HAM/TSP patients are usually below detectable levels [15]. Though CSF sIL-2R might be a useful indicator of CNS ATL, additional assessments are necessary for definitive diagnosis as it could be elevated by other diseases with active T-cell responses.

A pathological examination of brain tissue by biopsy or surgery is useful for a diagnosis if massive lesions are detected in the CNS. However, a brain biopsy is often inapplicable because of lesion size, anatomical location, and complication risks [19]. Alternatively, cytological analysis of CSF cells is essential, but discrimination of atypical and abnormal lymphocytes is often difficult in HTLV1-infected patients. Moreover, cytocentrifuge, which is generally used before the analysis, has been reported to induce morphological changes, and cytocentrifuged smears should be evaluated with caution [20]. Intriguingly, the sensitivity of CSF cytology alone is low (2-32\%), but the sensitivity increases when cytology is performed in combination with immunophenotypic analysis [21].

Immunophenotypic investigations on infiltrating cells in the CSF are valuable to confirm diagnosis in many cases ineligible for a brain biopsy. Typically, CSF samples of patients with HAM/TSP exhibit mild lymphocytic pleocytosis containing both CD4-positive HTLV-1-infected T-cells and CD8-positive cytotoxic cells targeting the HTLV-1 antigen [2]. In our case, there were few immune or inflammatory cells in the CSF, and most of the infiltrating cells in the CSF were immunophenotypically homogenous and strongly expressed CD4, CADM-1, and CCR4. Additionally, as few CD8-positive T-cells were seen in the CSF despite no use of an immunosuppressive agent, the HAM/TSP disease activity in this patient was considered to be low. The CD7 and CADM-1 expression pattern plotted for CD4-positive cells in the CSF was similar to the patterns often seen in patients with aggressive ATL [7-10]. By contrast, the expression pattern of CD4-positive cells in the PB was similar to the pattern observed in high-risk HTLV-1 carriers or indolent ATL patients [7-10]. This discrepancy between the CSF and PB was also supported by other clinical features found in this case, such as a normal level of serum LDH, no lymphocytosis, and fewer morphological abnormalities in peripheral lymphocytes. Based on these findings, we conclude that this patient newly developed aggressive ATL in the CNS, but the
HAM/TSP was not exacerbated. A flow-cytometric analysis of the CSF was quite useful for differentiating between ATL in the CNS and HAM.

We also reviewed the peripheral blood. Among the total WBCs $(6700 / \mu \mathrm{L}), 3 \%(201 / \mu \mathrm{L})$ of those were atypical lymphocytes, but no cells were classified as abnormal lymphocytes according to the standard guideline of the Japanese Society for Laboratory Hematology. Judged comprehensively with other clinical laboratory results presented above, the atypical cells in the PB would not be aggressive ATL cells. Recently we have reported CD4 + CADM1 + cell percentage in the $\mathrm{PB}$ predicts disease progression in HTLV-1 carriers and indolent ATL. The cases where the CADM $1+$ percentage exceeds $50 \%$ are unstable and at high risk of acute transformation. In such cases, the cumulative incidence of receiving systemic chemotherapy at 3 years was $28.4 \%$ [22]. Since the CADM1 + percentage, which is the total percentage of CD7D and CD7N in CD4-positive $\mathrm{T}$-cells, were as high as $57.7 \%$ in this case, the risk for progression to aggressive ATL was considered to be high. Immunophenotypic analysis is useful for evaluation of the risk, and the patient with high risk should have been monitored carefully.

Confirmation of monoclonal integration of HTLV-1 using southern blotting or inverse PCR is necessary for diagnosing aggressive ATL. Sequential analysis of the CSF and the $\mathrm{PB}$ is recommended for evaluating the disease activity of HAM/TSP and monitoring the risk of acute transformation. However, we were unfortunately unable to evaluate them due to difficulties in collecting enough samples and a short period of hospitalization.

In conclusion, we describe a case of a patient with HAM/ TSP who developed ATL rapidly in the CNS without systemic progression. When HAM patients present with unusual cranial nerve symptoms, a comprehensive investigation is necessary for an early diagnosis. Immunophenotypic characterization of the cell populations in the CSF and PB using multi-color flow cytometry was very valuable for making a differential diagnosis and understanding disease status.

\section{Compliance with ethical standards}

Conflict of interest The authors declare no conflict of interest.

\section{References}

1. Gessain A, Cassar O. Epidemiological aspects and world distribution of HTLV-1 infection. Front Microbiol. 2012;3:388.

2. Yamano Y, Sato T. Clinical pathophysiology of human T-lymphotropic virus-type 1-associated myelopathy/tropical spastic paraparesis. Front Microbiol. 2012;3:389. 
3. Nosaka K, Iwanaga M, Imaizumi Y, Ishitsuka K, Ishizawa K, Ishida Y, et al. Epidemiological and clinical features of adult T-cell leukemia-lymphoma in Japan, 2010-2011: a nationwide survey. Cancer Sci. 2017;108:2478-86.

4. Araya N, Sato T, Yagishita N, Ando H, Utsunomiya A, Jacobson $\mathrm{S}$, et al. Human T-lymphotropic virus type 1 (HTLV-1) and regulatory T cells in HTLV-1-associated neuroinflammatory disease. Viruses. 2011;3:1532-48.

5. Araya N, Sato T, Ando H, Tomaru U, Yoshida M, Coler-Reilly A, et al. HTLV-1 induces a Th1-like state in CD4 + CCR4 + T cells. J Clin Investig. 2014;124:3431-42.

6. Furukawa Y, Okadome T, Tara M, Niina K, Izumo S, Osame M. Human T-cell lymphotropic virus type-I (HTLV-I)-associated myelopathy/tropical spastic paraparesis with acute type of adult T-cell leukemia. Intern Med. 1995;34:1130-3.

7. Ishigaki T, Kobayashi S, Zaike Y, Watanabe E, Sato N, Ohno N, et al. Establishment of flow cytometric methods for evaluation of adult T-cell leukemia and their clinical application. Cytom Res. 2014;24(2):33-9 (Japanese).

8. Kobayashi S, Tian Y, Ohno N, Yuji K, Ishigaki T, Isobe M, et al. The CD3 versus CD7 plot in multicolor flow cytometry reflects progression of disease stage in patients infected with HTLV-I. PLoS ONE. 2013;8:e53728.

9. Kobayashi S, Nakano K, Watanabe E, Ishigaki T, Ohno N, Yuji $\mathrm{K}$, et al. CADM1 expression and stepwise downregulation of CD7 are closely associated with clonal expansion of HTLV-Iinfected cells in adult T-cell leukemia/lymphoma. Clin Cancer Res. 2014;20:2851-61.

10. Kobayashi S, Watanabe E, Ishigaki T, Ohno N, Yuji K, Nakano K, et al. Advanced human T-cell leukemia virus type 1 carriers and early-stage indolent adult T-cell leukemia-lymphoma are indistinguishable based on CADM1 positivity in flow cytometry. Cancer Sci. 2015;106:598-603.

11. Shimoyama M. Diagnostic criteria and classification of clinical subtypes of adult T-cell leukaemia-lymphoma. A report from the Lymphoma Study Group (1984-1987). Br J Haematol. 1991;79:428-43718.

12. Kitajima M, Korogi Y, Shigematsu Y, Liang L, Matsuoka M, Yamamoto T, et al. Central nervous system lesions in adult T-cell leukaemia: MRI and pathology. Neuroradiology. 2002;44:559-67.

13. Dungerwalla $M$, Osuji N, Waldman AD, Al Jehani F, Mehta A, Tailor R, et al. Isolated central nervous system involvement in adult T-cell lymphoma/leukaemia. Br J Haematol. 2005;130:511-5.

14. Tsukasaki K, Tobinai K. Clinical trials and treatment of ATL. Leuk Res Treat. 2012;2012:101754.

15. Sato T, Coler-Reilly A, Utsunomiya A, Araya N, Yagishita N, Ando H, et al. CSF CXCL10, CXCL9, and neopterin as candidate prognostic biomarkers for HTLV-1-associated myelopathy/tropical spastic paraparesis. PLoS Negl Trop Dis. 2013;7:e2479.

16. Sato T, Yagishita N, Tamaki K, Inoue E, Hasegawa D, Nagasaka $\mathrm{M}$, et al. Proposal of classification criteria for HTLV-1-associated myelopathy/tropical spastic paraparesis disease activity. Front Microbiol. 2018;9:1651.

17. van Westrhenen A, Smidt LCA, Seute T, Nierkens S, Stork ACJ, Minnema MC, et al. Diagnostic markers for CNS lymphoma in blood and cerebrospinal fluid: a systematic review. Br J Haematol. 2018;182(3):384-403.

18. Uozumi K, Ishitsuka K, Ohno N, Nakahara K, Utsunomiya A, Hanada S, et al. Significance of elevated levels of soluble factors in the cerebrospinal fluid in patients with adult T-cell leukemia. Leuk Lymphoma. 1995;19:437-45.

19. Haldorsen IS, Espeland A, Larsson EM. Central nervous system lymphoma: characteristic findings on traditional and advanced imaging. AJNR Am J Neuroradiol. 2011;32:984-92.

20. Hu X, Laguerre V, Packert D, Nakasone A, Moscinski L. A simple and efficient method for preparing cell slides and staining without using cytocentrifuge and cytoclips. Int J Cell Biol. 2015;2015:813216.

21. Scott BJ, Douglas VC, Tihan T, Rubenstein JL, Josephson SA. A systematic approach to the diagnosis of suspected central nervous system lymphoma. JAMA Neurol. 2013;70(3):311-9.

22. Makiyama J, Kobayashi S, Watanabe E, Ishigaki T, Kawamata $\mathrm{T}$, et al. CD4 + CADM1 + cell percentage predicts disease progression in HTLV-1 carriers and indolent adult T-cell leukemia/ lymphoma. Cancer Sci. 2019;110(12):3746-53.

Publisher's Note Springer Nature remains neutral with regard to jurisdictional claims in published maps and institutional affiliations. 